\title{
Ultrasound microbubble contrast agent - carried chemotherapeutic drug microbubbles
}

\author{
Junyang Song ${ }^{1, \mathrm{a}}$ \\ ${ }^{1}$ Shaanxi University of Chinese Medicine, Second Clinical Medicine College Clinical Medicine Department, 712046 Shaanxi Xianyang, \\ China
}

\begin{abstract}
With the continuous research and development of ultrasound microbubble contrast agent-carried chemotherapeutic drug microbubbles, ultrasound microbubble contrast agent not only facilitates the early detection and early diagnosis of tumors but also provides a new direction and development prospect for the drug delivery route. With a broad development prospect, it is expected to become a new safe, effective and non-invasive treatment. This paper reviews the biological effects and research progress of contrastenhanced microbubble-loaded chemotherapy drugs in tumor therapy.
\end{abstract}

\section{The biological effects of ultrasound microbubbles on tumor}

\subsection{Mediating tumor cell apoptosis}

The cavitation effect refers to the microbubble contrast agent accumulating energy in the microbubbles under the action of ultrasound. When the energy exceeds a certain threshold, the microbubbles rupture, generating energy and shock waves and destroying the surrounding tissue. Cavitation and thermal effects can make tumor cells highly sensitive to chemotherapeutic drugs and promote the killing of tumor tissue cells[1,2]. Lowfrequency and low-power ultrasound technology combined with ultrasonic microbubbles can enhance the killing effect of ultrasound on tumor cells through cavitation effect and sonoporation effect. The sonoporation effect refers to the destruction of tumor cell membranes on the basis of cavitation effect and then produce hole. Studies have found that the mechanism of tumor cell apoptosis may be low-frequency ultrasound can promote the production of reactive oxygen species. With the increase of reactive oxygen species, different ways to induce tumor cell apoptosis. In addition,lowfrequency ultrasound can also weaken the activity of superoxide dismutase, reduce the ability of eliminating oxygen free radicals, and increase the activity of reactive oxygen species. Compared with normal tissue cells, tumor tissue cells are more susceptible to killing under ultrasound irradiation, indicating that ultrasound has a higher therapeutic sensitivity to tumor cells and can effectively kill tumor cells without damaging normal cells.

\subsection{Destruction or congestion of tumor nourish blood vessels}

The production of tumor blood vessels provides the necessary conditions for the growth and metastasis of tumor cells. Therefore, it can effectively prevent the growth and metastasis of tumors by destroying or clogging tumors to nourish blood vessels and has achieved good clinical results. In this respect, ultrasound contrast microbubbles can destroy tumor nourishment vessels, or block tumor blood vessels to induce tumor cell apoptosis. The drug for embolizing the tumor is integrated into the ultrasound microbubble to act on the tumor tissue by exciting the coagulation waterfall, but it is required that the prothrombin stays in the blood vessel of the tumor tissue and does not affect the vascular bed in the normal tissue. The study found that ultrasound microbubbles have the potential to accomplish this task. Tumor vascular embolization targets can use growth factor receptors and adhesion molecule family receptors of neovascular endothelial cells, and this target is expected to achieve targeted therapy of tumors.

\subsection{Mediated transport of chemotherapeutic drugs or genes}

At present, an important method for treating tumors is chemotherapy, but the chemotherapeutic drugs are not targeted, which causes the chemotherapeutic drugs to destroy the normal tissue cells while acting on the tumor tissue cells, and it is difficult to avoid toxic side effects. In recent years, research has continuously found that ultrasound microbubbles containing chemotherapeutic drugs are promising as a new mode of administration. Ultrasound microbubbles can be selectively targeted to specific sites after being modified in different ways. 
Ultrasound microbubbles provide a new methods for targeted transportation of chemotherapeutic drugs and brings a new directions for cancer treatment.The combination of contrast-enhanced microbubbles and chemotherapeutic drugs is as follows: 1 . Chemotherapeutic drug directly adheres to the microbubble shell; 2 . Encapsulating the chemotherapeutic drug in the microbubble; 3 . Chemotherapeutic drug forms a non-covalent bond with the microbubble shell; 4. If the contrast-enhanced microbubbles are bilayer shells, chemotherapy drugs can be placed between the inner shells of the shells. Ultrasound technology is combined with drug-loaded microbubbles to release chemotherapeutic drugs in tumor tissues through cavitation effects, promoting the delivery of chemotherapeutic drugs into tumor tissues through sonication, and avoiding damage to other normal tissues while killing tumor cells. The method not only reduces the toxicity of the chemotherapy drug but also improves the utilization rate of the drug.

Today, gene therapy is the most promising way to treat malignant tumors. Gene-targeted therapy for tumors inhibits tumor growth by transfecting genes that cause apoptosis and growth inhibition. The sonication effect of ultrasound microbubbles can generally increase the transfection rate of tumor cells and improve the efficacy of tumor gene-targeting therapy. The key to the success of gene-targeted therapy is the efficient transfection of foreign genes. The conditions for efficient gene transfection are changes in cell membrane permeability. In recent years, a study[3] found that ultrasound contrast microbubbles can not only reduce the cavitation threshold of ultrasound but also enhance the cavitation effect, further promoting the entry of plasmid DNA into the cell, thereby increasing the transfection rate of the gene. Guo et al. [4] observed that ultrasound irradiation combined with contrast microbubbles could express exogenous low-density lipoprotein receptor gene at a greater level through hepatocyte gene transfection experiments.

\section{Research of contrast-enhanced microbubbles in tumor treatment}

\subsection{Ultrasound-mediated research of drug- loaded microvesicles carrying chemotherapy drugs in liver cancer}

At present, the main methods for treating liver cancer include surgery, chemotherapy, radiotherapy, targeted therapy,and immunotherapy. Chemotherapy is an important method for the treatment of liver cancer, which can effectively prolong the survival time of patients and effectively improve the quality of life of patients. However, chemotherapy drugs lack a safe and effective drug delivery channel, and have large side effects, causing irreversible damage to normal human cells. Adverse reactions limit its clinical application. With the continuous development and research of ultrasound imaging technology, ultrasound-targeted microbubble destruction (UTMD) technology can mediate drug-loaded microbubble targeted release drugs, providing a new mode of drug delivery for tumor chemotherapy [5], which has received extensive attention from scholars at home and abroad.

Targeted therapy for liver cancer needs to meet two conditions. Firstly, the specific delivery of chemotherapeutic drugs to tumor tissue sites, and secondly, promoting the uptake of chemotherapeutic drugs by tumor cells. The high uptake of chemotherapeutic drugs by tumor cells is the key to the efficacy of chemotherapeutic drugs[6]. Ultrasoundmediated drug-loaded microbubbles can effectively meet these two conditions.Ultrasound microbubbles carry chemotherapeutic drugs and target liver cancer tissues. They can store high concentrations of chemotherapeutic drugs in tumor tissues, prolong the killing time of tumor cells, and reduce the side effects of other normal tissues. Ultrasound microbubbles can be used as carriers to carry chemotherapeutic drugs to cancerous tissues. When the microbubbles of the drug-loaded microbubbles are under the cavitation and sonoporation effects of ultrasound [7], the microbubbles change. When the acoustic wave can reach a certain strength, the microbubbles will break in the tumor, and the chemotherapy drugs will be released to the tumor site, reducing the toxic side effects of the drug on normal tissues.

Paclitaxel(PTX) is currently one of the commonly used chemotherapy drugs in the clinic, but PTX has many serious adverse reactions. Studies have found that PTX-loaded lipid microbubbles significantly reduced the side effects of paclitaxel on normal cell tissue. A study have found that HepG2 cells can be induced by PTX to induce apoptosis, but after irradiation with PTX-loaded microvesicles on the tumor site, the apoptosis rate of tumor cells is higher and prolonged with time.The inhibitory effect on liver cancer is also enhanced. Hayashkla et al. [8] mixed carbon dioxide microbubbles with absolute alcohol, after treatment can induce tumor necrosis less than $3 \mathrm{~cm}$ in diameter, and no other locations such as intrahepatic metastasis were found. Kang et al. [9] found that ultrasound-targeted microbubble destruction technology can significantly inhibit the growth of rabbit VX2 hepatoma cells,promote the apoptosis of liver cancer cells, and inhibit the growth of liver cancer, prevent the metastasis of tumor cells, and improve the survival rate. The above studies have greatly demonstrated the safety and targeting of ultrasonic drugloaded microbubbles in liver cancer, greatly reducing the adverse reactions of chemotherapy drugs, and providing a new mode of administration for the treatment of liver cancer.

\subsection{Ultrasound-mediated research on drug- loaded microvesicles carrying chemotherapy drugs in related aspects of female malignant tumors}

Breast cancer and ovarian cancer are the two most common tumors in women with primary malignant tumors. Currently, the treatment of breast cancer and ovarian cancer is mainly based on traditional surgical 
treatment, chemotherapy and radiotherapy. It is often difficult to cure and is prone to recurrence. Therefore, it is urgent to seek a safer and more effective treatment. The study found that ultrasound-mediated drug-loaded microbubbles as a new mode of administration, promising for targeted therapy of breast and ovarian cancer, provides a safer and more effective drug treatment for patients with breast and ovarian cancer.

Rapoport et al. [10] found that breast cancer cells can be ultrasound-mediated inhibition of paclitaxel microbubbles, and the tumor volume is significantly reduced after administration. LYP-1 peptide is a homing peptide of breast tumor. This peptide is a cyclic nonapeptide molecule obtained by phage screening. The P32 receptor overexpressed on the surface of tumor cells and tumor lymphatic endothelial cells can be specifically Sexual recognition $[11,12]$. Studies have reported that LYP-1 peptide binds to paclitaxel-loaded microbubbles and can be targeted to MDA-MB-231 human breast cancer cells. The effective absorption rate of cells using ultrasound-loaded microvesicles is no ultrasound microbubbles. Several times the group [13]. Ultrasound combined with drug-loaded microbubbles enhances the anti-tumor ability of paclitaxel and can be used as a safe and effective chemotherapy for breast cancer.

Rapoport et al. [14] used polymer micelles and perfuloron-pentane to prepare doxorubicin-carrying nanoscale ultrasound microbubbles to treat nude mice with ovarian cancer and drug sensitivity, and found nanoscale ultrasonic microbubbles can enter the tumor cells in a targeted manner, and hardly stays in other normal tissues and organs, and has a significant repression effect on tumor growth. PLGA-PTX is a new type of polymer-drug form that is biodegradable and has high encapsulation efficiency and drug loading rate.In recent years, with the advancement of targeted ultrasound microbubbles, luteinizing hormone releasing hormone (LHRH) receptors can be highly expressed on ovarian cancer cells, such as Liu et al. [15], using this property on PTX ultrasound microbubbles.Fusion of luteinizing hormone-releasing hormone analog(LHRHa), through the biotin-linked ovarian cancer A2780/DDP cells to express LHRH receptor, so as to achieve the purpose of targeted drug delivery. The experimental results showed that the LHRHa-targeted MBs group had a much higher inhibitory effect on ovarian cancer than the other groups. The LHRHa-targeted pEGFP-N1wild-type p53 microvesicles produced by Chang et al. [16] were transfected into A2780/DDP cells, and the transfection rate and apoptosis rate of the targeted ultrasound microbubble group were higher than those of the other treatment groups.

\subsection{Ultrasound-mediated research of drug- loaded microvesicles carrying chemotherapy drugs in intracranial tumors}

The most common primary malignant tumor in the central nervous system is glioma. Because of the presence of the blood-brain barrier (BBB), it is currently clinically less effective in treating gliomas and has a lower survival rate. In recent years, studies have found that ultrasound contrast microbubbles can penetrate the blood-brain barrier non-invasively and effectively, bringing new ways of administration for the treatment of glioma. The transferrin receptor (TfR) can be highly expressed on the glioma cell membrane, and the transferrin receptor-binding peptide can specifically bind to the glioma cell membrane TfR. A Chinese scholar combined ultrasound microbubbles and binding peptides. Ultrasound microbubbles can safely and effectively promote the drug to pass through the $\mathrm{BBB}$, and release it at the tumor tissue. The peptide binds the drug to the glioma cells. It is selective and ultimately achieves targeted therapy for glioma. With the study of microbubbles of ultrasound microbubble-loaded chemotherapy drugs, ultrasound microbubbles can effectively open the blood-brain barrier and deliver chemotherapy drugs to tumor tissues to treat intracranial tumors, providing a new treatment for intracranial tumors.

\section{Insufficient and prospect of ultrasound contrast microbubbles in application}

At present, the contrast-enhanced microbubbles in the delivery of chemotherapy drugs exist: 1. Microbubble stability is not high; 2 . Ultrasound contrast microbubbles carrying drugs or poor gene efficiency; 3. Poor targeting; 4. Cause tissue hemorrhage, injury and other adverse reactions and so on, but the benefits are still far greater than the drawbacks. Ultrasonic microbubble contrast agents can be divided into albumin, nonionic surfactants, liposomes, and polymer materials after years of development according to the material of the membrane. At present, the contrast-enhanced microbubbles have been experienced for four generations. The firstgeneration ultrasound microbubble contrast agent is mainly represented by human serum albuminencapsulated air. The second-generation ultrasound microbubble contrast agent is characterized by the introduction of sulfur hexafluoride and perfluocarbon, which are high molecular weight gas, and membrane material began to transform into liposomes. The third-generation ultrasound contrast microbubbles began to be made of high molecular polymer materials. The fourth-generation ultrasound contrast microbubbles have a targeting function. Ultrasonic microbubble contrast agents have become more and more targeted through continuous development and application, but there are still problems in stability and so on.

For the study of contrast-enhanced microbubbles on tumors, in addition to being used as a drug-loading tool to target chemotherapeutic drugs to tumor tissues, it can also destroy tumor nourishing blood vessels to treat cancer. At present, vascular embolization of tumor tissue and tumor vascular rupture to treat tumor is a new treatment method for cancer after radiotherapy, radiotherapy and surgery, but it can only be used for large blood vessels due to limitations of technology and equipment. Some scholars applied ultrasound-mediated 
microbubbles to rupture tumor nourishment vessels and cause tumor degeneration, thus preventing tumor growth, which is likely to become a kind of damage caused by the use of ultrasound microbubbles without the need to add chemotherapy drugs. A more effective method for tumors to nourish blood vessel rupture to inhibit tumor growth. At present, the two methods of using ultrasound contrast microbubbles to treat tumors are still under study and have great development prospects. Both methods have their own shortcomings and advantages. Here, I suspect that the combination of the chemotherapeutic drug contrast microbubbles and the tumor vascular rupture contrast microbubbles can be combined to target tumor tissue, which may better inhibit tumor cell growth and metastasis and improve patient survival.

\section{Summary}

In summary, ultrasound contrast microbubbles are still in the research stage in the delivery of chemotherapy drugs, and still need to be continuously explored. Therefore, the development of new contrast agents that can more effectively bind and encapsulate genes or drugs is the future development direction. Recently, a new type of nanoscale ultrasonic microbubble contrast agent has emerged, which has better penetrability and higher stability than current micron-sized ultrasound contrast microbubbles, and has great potential in targeted therapy.

\section{References}

1. H. Ashush, L. A. Rozenszajn, M. Blass, Cancer Res, 60, 1014 (2000)

2. D. L. Miller, S. V. Pislaru, J. E. Greenleaf, Somat Cells Mol Genet, 27, 115(2002)

3. R. Bkeredjian, C. Bohris, A. Hansen, Ultrasound Med Biol, 33, 743(2007)

4. D. P. Guo, X. Y. Li, P. Sun, BiochemBiophys Res Commun, 343, 470(2006)

5. F. Kiessling, S. Fokong, P. Koczera, J Nucl Med, 53, 345(2012)

6. N. Nomikou, A. P. McHale, Cancer Lett, 296, 133(2010)

7. A. C. Ward, Biochen Soc Trans, 27, 128(1999)

8. K. Hayashida, J. Ooi, K.Omagari, Nippon Shokakibyo Gakkai Zasshi, 94, 730(1997)

9. J. Kang, X. Wu, Z. Wang, J Ultrasound Med, 29, 61(2010)

10. N. Y. Rapoport, A. M. Kennedy, J. E. Shea, J Control Release, 138, 268(2009)

11. P. Laakkonen, M. E. Akerman, H. Biliran, Proc Natl Acad Sci USA, 101, 9381(2004)

12. V. Fogal, L. Zhang, S. Krajewski, Cancer Res, 68, 7210(2008)

13. F. Yan, X. Li, Q. Jin, Ultrasound Med Biol, 37, $768(2011)$
14. N. Rapoport, Z. Gao, A. Kennedy, J Natl Cancer Inst, 99, 1095(2007)

15. H. Liu, S. Chang, J. Sun, Mol Pharm, 110, 40(2014)

16. S. Chang, J. Guo, J. Sun, UltrasonSonochem, 20, 171(2013) 